\title{
Raman System for Sensitive and Selective Identification of Volatile Organic Compounds
}

Kelly J. Park ${ }^{1}$, Constance Wu ${ }^{1}$, Alison R. Mercer-Smith ${ }^{1}$, Ryan A. Dodson ${ }^{1}$, Tyler L. Moersch ${ }^{1}$, Prakash Koonath ${ }^{2}$, Andrew C. R. Pipino ${ }^{2}$, Hsiang-Wei Lu ${ }^{3}$, Yongwu Yang ${ }^{4}$, Victor S. Sapirstein ${ }^{4}$, Charles J. Taylor ${ }^{1}$, Angelika Niemz ${ }^{3}$ *

${ }^{1}$ Chemistry Department, Pomona College, 645 N. College Avenue, Claremont, California 91711

${ }^{2}$ Tanner Research Inc., 825 South Myrtle Ave., Monrovia, CA 91016

${ }^{3}$ Keck Graduate Institute of Applied Life Sciences, 535 Watson Drive, Claremont, CA 91711

${ }^{4}$ Lambda Solutions Inc., 411 Waverly Oaks Road, Waltham, MA 02452

*aniemz@kgi.edu

Keck Graduate Institute of Applied Life Sciences, 535 Watson Drive, Claremont, CA 91711.

Tel.: (909) 607-9854 | Fax: (909) 607-9826

Current affiliations: K.J. Park: Food and Drug Administration, 1 World Trade Center, Ste 300, Long Beach, CA 90831; C. Wu: Department of Biochemistry, University of Cambridge, 80 Tennis Court Road, Old Addenbrooke's Site, Cambridge CB2 1QW, UK; T.L. Moersch: Tru Vue, Inc., 2150 Airport Drive, Faribault, Minnesota 55021; A.C.R. Pipino: Office of Naval Research, 875 N. Randolph St, Arlington, VA 22203

Keywords: VOC, Raman, 2D Correlation Analysis, Polymer thin film, Biomarker, Evanescent wave 


\begin{abstract}
Analysis of volatile organic compounds (VOCs) for medical and industrial applications typically requires complex and expensive mass spectrometric systems to achieve the desired sensitivity and specificity. Raman spectroscopy enables specific compound identification based on distinct spectral fingerprints but traditionally has low sensitivity. We have developed a novel Raman system that provides VOC detection in the low ppm range. VOCs from the gas phase are absorbed and enriched in a thin polymer film coated on the surface of an optical sensor element. The VOC enriched polymer film is probed with evanescent wave excitation and optimized Raman signal collection. The herein described Raman sensor is low-cost, robust, readily manufacturable, and capable of providing high sensitivity without the nanostructured sensors required for surface enhanced Raman spectroscopy. To suppress background noise and interfering spectral features while improving our ability to resolve VOC mixture spectra, we applied two-dimensional (2D) correlation analysis to time series spectra acquired during VOC absorption into the polymer film. We established proof of principle through analysis of known VOC biomarkers for selected bacterial pathogens. The system enabled VOC detection with significantly higher sensitivity than commercial Raman probes. We observed good agreement between spectra of VOCs in the thin film obtained after 2D correlation analysis, and of pure VOCs using a commercial probe. Mixture spectra were further de-convoluted based on the offdiagonal peaks observable in the 2D Raman spectrum in conjunction with segmented data analysis. We anticipate that this system can be applied to a variety of medical, industrial, and biodefense applications.
\end{abstract}




\section{Introduction}

Analysis of volatile organic compounds (VOCs) from breath or the headspace of liquid culture, urine, blood, stool, or sputum can be used to identify pathogenic bacteria and fungi, to detect cancer cells, and to diagnose metabolic disorders [1-14]. Even though mass spectrometric techniques are the gold standard for VOC biomarker identification, these systems are complex and expensive and therefore have not emerged for routine clinical use. However, breath analyzers to determine alcohol intoxication levels [15-17], Helicobacter pylori infection [16,18], and airway inflammation [19-21] are routinely used in point-of-care or point-of-use diagnostic systems. These devices use electrochemistry [17,19,21] or infrared (IR) spectroscopy $[15,16,18,20]$ to detect simple single volatile compounds, and while these systems have reduced instrumental complexity, they cannot analyze complex VOC mixtures at lower abundance. Electronic noses (eNoses) and colorimetric sensor arrays can analyze VOC mixtures at low abundance using multiple relatively non-specific chemical sensors [10,22-25]. However, eNoses measure an aggregate response to VOC mixtures, and not the mixture composition. Furthermore, eNose sensor arrays typically require elaborate calibration and cannot be readily adapted to new or revised VOC signatures.

Raman spectroscopy can identify chemical compounds with high selectivity because a unique spectral fingerprint exists for each molecule. Compared to IR spectra, Raman spectra have sharper features, enabling higher selectivity, and are more resistant to water interference. However, conventional Raman spectroscopy is not very sensitive. Sensitivity can be enhanced by many orders of magnitude using surface-enhanced Raman spectroscopy (SERS) [26-28], but SERS requires nano-structured substrates, which are fragile and difficult to manufacture in a manner that ensures reproducible results. On the other hand, evanescent wave Raman excitation 
can be used to analyze compounds at or near a surface with increased sensitivity by exploiting the two-photon character of normal Raman scattering, together with the surface electric field enhancement. Robust solid immersion lenses (SIL), fabricated from high refractive index sapphire or ZnSe, enable exceptionally high evanescent excitation and collection efficiency [29,30]. Compared to IR absorption, evanescent-wave Raman derives a stronger signal enhancement from total internal reflection (TIR) because the TIR electric field enhancement is approximately squared in the Raman cross-section, as both the excitation and Raman-shifted photons experience a comparable enhancement [31]. Evanescent wave TIR Raman has been employed to probe thin polymer films $[30,32,33]$ and the structure of water near LangmuirBlodgett films [34].

Attenuated total reflection (ATR)-IR sensors have been used to measure traces of organic compounds in water, mainly for environmental applications [35-43]. These systems employ a thin polymer film $[38,40-42]$ or sol gel layer $[39,43]$ to absorb organic compounds, concentrating the analyte in the evanescence sensing region, and enabling limits of detection in the low ppm and in some cases ppb range. However, these types of sensors have not yet been applied to measuring VOCs in air. Furthermore, resolving multi-component mixtures with overlapping bands in a spectrum is challenging and limits the specificity of the sensor.

Two-dimensional (2D) correlational spectroscopy is a powerful technique used to extract information about spectral bands changed by a perturbation from a plethora of spectral features insensitive to change [44,45]. It is routinely used in nuclear magnetic resonance (NMR) spectroscopy and has also been extensively applied to vibrational spectroscopy [44-48]. The correlation transform provides one dimensional (1D) spectra from which background features are effectively removed. Overlapping spectral features can be spread out in the 2D plane, permitting 
analysis of complex mixtures [45,46,49]. 2D-correlation Raman spectroscopy involves the collection of a set of 1D Raman spectra from a system in response to a perturbation. The collected spectra are then subjected to 2-D correlation analysis, resulting in the so-called synchronous and asynchronous spectra [45]. Sets of peaks that change during time series data acquisition with identical time constants either perfectly in phase or $90^{\circ}$ out of phase will only have cross-peaks in the synchronous or asynchronous 2D spectrum, respectively. Most practical cases are in-between these two extremes, and thus will give rise to cross-peaks in both spectra, but with different relative intensities. In 2D spectroscopy, any spectral feature that does not change with the perturbation vanishes from the final result. 2D-correlation IR spectroscopy has been applied to study water absorption into polymer films [50,51], adsorption of small molecules at interfaces [52], and diffusion of small molecules inside polymers [53,54], but has not been applied to the types of ATR-IR sensors mentioned above. 2D-correlation Raman analysis has been used to study a wide range of compounds including small molecules, polymers, proteins, lipids, and DNA [48,55-59]. However, to the best of our knowledge, there have been no previous demonstrations of evanescent wave Raman spectroscopy on polymer films enriched with organic compounds from aqueous solution or the gas phase, and of the analysis of their spectra via 2-D correlation analysis.

In this study, we present proof of principle for a novel Raman system that enables VOC analysis from the gas phase. This system entails a robust optical sensor design using commercial off-the-shelf components appropriate for mass production. VOC enrichment in a thin polymer film, combined with evanescent wave excitation and optimized signal collection, provides sensitivity much higher than existing commercial Raman probes and comparable to similar ATRIR sensor designs. Specific identification of individual VOCs and the ability to resolve mixture 
spectra are facilitated by the inherently sharp features of Raman spectroscopy, coupled with 2D correlation analysis. We anticipate that this system, once suitably refined, can be applied to different medical, industrial, and biodefense applications. 


\section{Experimental}

\section{Optical Design}

The optical system used in these studies consists of a Dimension P2 Raman spectrometer (Lambda Solutions Inc., Waltham, MA) in conjunction with a custom designed sensor with evanescent wave excitation, as described in Figure 1. The sensor surface consists of the flat side of a $10 \mathrm{~mm}$ diameter ZnSe hemisphere lens (ISP optics, Irvington, NY), coated with a thin polymer film. This hemisphere was mounted above an aspheric lens $(\mathrm{NA}=0.86,30 \mathrm{~mm}$ diameter). As shown in Figure 1 (top), the excitation laser beam is introduced into the optical path by reflection from a 0.5 " diameter gold-coated mirror and from a custom dichroic filter (transmission band $=849.1-1286.5 \mathrm{~nm}$, Semrock), which is rotated by $45^{\circ}$ relative to the optical axis. The angle-of-incidence at the TIR surface of the ZnSe hemisphere was controlled via the position of the laser beam reflection on the dichroic mirror, which in turn is determined by diagonal translation of the gold-coated mirror mounted on a micrometer-controlled linear translation stage. To enable vertical orientation of the sensing element, the incident beam was reflected by $90^{\circ}$ from another mirror into the aspheric lens and $\mathrm{ZnSe}$ hemisphere (not shown in Figure 1, top). The hemisphere - asphere separation was adjusted by a pair of micrometer screws installed in the plate holding the $\mathrm{ZnSe}$ hemisphere. A custom flow cell with Luer lock connectors on the inlet and outlet was mounted on top of the plate holding the ZnSe hemisphere with a custom Teflon gasket to ensure an air-tight chamber.

\section{Thin Film Deposition and Characterization}

A commercial lens coating and cleaning solution (First Contact ${ }^{\circledR}$, Photonic Cleaning Technologies, Platteville, WI) was used to prepare polymer thin films for the experiments reported herein. The First Contact ${ }^{\circledR}$ solution consists of a proprietary polymer blend dissolved in 
a solvent mixture. Thin films were drop cast, by depositing approximately $10 \mu \mathrm{L}$ of this solution onto the center of the flat $\mathrm{ZnSe}$ lens surface, and allowed to cure at room temperature for at least 1 hour. Polymer films were removed from the lens after each experiment, resulting in a clean surface.

To characterize the film thickness, ten First Contact ${ }^{\circledR}$ films were deposited in the same manner on glass slides. These films were then analyzed by stylus profilometry using a XP-1 stylus profiler (Ambios /KLA-tencor, Milpitas, California). Films were also imaged before and after the profilometry scans using an Olympus BH-2 light microscope under bright field illumination.

\section{Raman Data Acquisition}

Raman spectra were acquired using a Dimension P2 Raman spectrometer (Lambda Solutions Inc.), with laser excitation at $830 \mathrm{~nm}$, integration time from 0.5 to $3 \mathrm{sec}$ unless otherwise noted, and 10 frames averaged per spectrum. To characterize the polymer background, spectra were acquired for the First Contact ${ }^{\circledR}$ polymer, in bulk and as a thin film. To provide reference spectra for the VOC biomarkers isovaleric acid, 2-aminoacetophenone, ethyl acetate, and indole (Sigma Aldrich), spectra were acquired of each compound as pure liquid, or solid in the case of indole, at room temperature. Furthermore, spectra were acquired for these VOCs absorbed from the gas phase into the First Contact ${ }^{\circledR}$ polymer thin film at room temperature. Bulk spectra were acquired using an LSI Raman Vector Probe (Lambda Solutions, Inc.), while the setup shown in Figure 1 was used for evanescent wave thin film experiments.

The small molecule organic compounds were brought into the gas phase and introduced into the flow cell in the following manner: for single compound experiments, $25 \mu \mathrm{L}$ of isovaleric 
acid, 2-aminoacetophenone, or ethyl acetate were deposited into a $13 \mathrm{~mm}$ diameter PTFE syringe filter. For mixture experiments, equal masses of these compounds were combined, and $25 \mu \mathrm{L}$ of this mixture was deposited into the syringe filter. For indole, 0.5-1 $\mathrm{mg}$ solid compound was deposited into a syringe filter wetted with deionized water. This was done to retain the solid powder on the filter. The solubility of indole in water is $3.56 \mathrm{~g} / \mathrm{L}$ at $25^{\circ} \mathrm{C}$ [60]. Therefore the amount of indole dissolved in the water on the filter is negligible, and the indole can be regarded as bulk solid. The so treated syringe filter was then attached to the inlet port of the flow cell. Medical grade compressed air was passed through the filter at a rate of $3.25 \mathrm{sccm}$ using a mass flow controller, exposing the polymer-coated ZnSe lens surface to a stream of gas-phase analyte. The gas phase concentration for each analyte was estimated based on the analyte's vapor pressure, as further detailed in the Supplementary Material. The calculated gas phase concentrations are estimates, representing the maximum value. The actual VOC gas phase concentrations are likely lower than this estimate.

For VOC uptake experiments into the polymer, Raman spectra were collected over a 1-hour period at 30 -second intervals in most cases. To estimate the attainable signal enhancement, a thin polymer film was saturated with 2-aminoacetophenone by passing this compound over the polymer film in the flow-cell for at least 5 days. Spectra of this film were then acquired using the evanescent-wave Raman probe and the commercial Raman Vector probe, which was mounted on an XYZ stage to enable precise focusing of the probe beam onto the film.

\section{Data Analysis}

The collected time series Raman spectral data were first processed to remove background and suppress noise. Background removal was performed using a method proposed by Zhao et al. [61], wherein a polynomial fitting algorithm is used to derive a background function for each 
spectrum, which is then subtracted from the original spectrum (see Supplementary Material). Noise removal was performed via principal component analysis (PCA) using a method described by Jung [62]. This PCA method identifies important factors governing the data structure and rejects noise components, as explained further in the Supplementary Material. The processed time series Raman spectral data were then analyzed via 2D correlation spectroscopy [45]. The algorithms for background subtraction, PCA-based noise removal, and 2D correlation spectroscopy analysis were implemented in MATLAB.

\section{Results and Discussion}

The key optical component of the sensor system is a small $\mathrm{ZnSe}$ hemisphere with high refractive index (Figure 2), which enhances the evanescent wave excitation intensity and the directive emission of the Raman signal. Furthermore, ZnSe produces no background Raman peaks in the region of interest, as opposed to other high refractive index optical sensor materials such as sapphire. The hemispherical $\mathrm{ZnSe}$ optic, combined with a high numerical aperture aspheric lens, enables most of the Raman emission to be collected, thereby providing enhanced sensitivity.

To demonstrate VOC enrichment in the evanescent wave region, we selected a commercially available lens coating film (First Contact ${ }^{\circledR}$ ) which efficiently absorbs and enriches VOCs, can be readily applied and removed from the sensor lens, and forms reasonably uniform films of appropriate thickness to enable evanescent excitation exclusively inside the polymer film. We prepared thin films by drop casting First Contact ${ }^{\circledR}$ polymer solution onto the $\mathrm{ZnSe}$ lens. This process results in a circular film $\sim 4 \mathrm{~mm}$ in diameter at the center of the $10 \mathrm{~mm}$ diameter lens. Profilometry experiments of ten films prepared in an analogous manner on glass slides (see Supplementary Material, Figure S1) indicated that the film thickness varied from film to film, 
but that each film was reasonably uniform in the region probed by the laser. In the region \pm 1 $\mathrm{mm}$ from the center of the film, the average film height was $73 \mu \mathrm{m}$, with a standard deviation of $\pm 31 \mu \mathrm{m}$ between films. For each film, the standard deviation of the film height in this center region was approximately $4 \mu \mathrm{m}$. Optical imaging revealed that most films were uniform, but 3 out of 10 films contained either small bubbles or other deformations. None of the films were damaged by the profilometry scans.

Evanescent field excitation probes a thin layer at the interface from which the incident light is reflected. The penetration depth of the evanescent field depends on the excitation wavelength, interfacial refractive indices, and angle of incidence [64]. In our system we estimate a penetration depth of $\sim 1 \mu \mathrm{m}$, which is much smaller than the polymer film thickness in the region probed by the laser. Therefore, only compounds that diffuse through the polymer film and reach the interface are interrogated. Significant variations in film height however can impact the diffusion time of VOCs through the film and into the evanescent region. Therefore, the thin film preparation process will be optimized in future efforts to obtain more reproducible and controllable film thicknesses and uniform films. Furthermore, variability in absorption kinetics can be can be taken into account by selecting a suitable time interval for 2D correlation analysis.

To collect Raman spectra with evanescence mode excitation, example VOCs were introduced via a stream of air into a flow cell mounted on top of the stage holding the ZnSe optic (Figure 1e). In these experiments, we found that the intensity of signal and background are critically dependent on the distance between hemisphere and asphere and on the excitation laser's angle of incidence $\Theta$ relative to the surface normal of the hemisphere (Figure 2). The asphere-hemisphere distance was tuned such that the hemisphere surface lies in the focal plane of the asphere to ensure proper optical alignment. The angle of incidence is controlled by the position of the gold 
mirror (Figure 1b). Evanescent excitation requires the excitation laser's incident angle to be equal to or greater than the critical angle. In the experiments described herein, we adjusted the asphere-hemisphere distance and the excitation laser's angle of incidence to provide optimal signal-to-background ratios and dynamic range.

Using this experimental setup, we then acquired Raman spectra for validated VOC biomarkers reported in the literature $[1,2,65,66]$ for identification of P.aeruginosa (2aminoacetophenone and 2-butanone), S.aureus (isovaleric acid and 2-methyl butanal), and E.coli (indole and ethyl acetate). Figure 3 shows a data set for isovaleric acid, a VOC biomarker for S.aureus. In this experiment, isovaleric acid in the gas phase was introduced via a stream of air into the flow cell at a concentration of at most $617 \mathrm{ppm}_{\mathrm{v}}$ (see Supplementary Material). A series of spectra were acquired in $0.5 \mathrm{~min}$ intervals over 1 hour. The obtained spectra consist of a combination of the Raman signatures of the polymer (see Supplemental Material, Figure S2) and the VOC absorbed into the polymer (Figure 3b). At wavenumbers corresponding to Raman emission bands of isovaleric acid (e.g. peaks at 836 and $962 \mathrm{~cm}^{-1}$, Figure 3a and b), the signal intensities increase as function of time. In contrast, spectral bands of the polymer remain relatively unchanged, except for a negative baseline drift (e.g. at $810 \mathrm{~cm}^{-1}$, Figure $3 \mathrm{a}$ and b).

The time series of spectra were then background subtracted using a polynomial fitting algorithm [61], followed by noise removal via PCA [62]. Next, the corrected time series spectra were subjected to $2 \mathrm{D}$ correlational analysis, a powerful technique that can identify spectral bands that change based on a perturbation. In this case, the perturbation consists of VOC uptake into the polymer as function of time, dictated by the diffusion coefficient of the VOC in the polymer. Other spectral features insensitive to change (in this case the polymer bands) are effectively suppressed. Correlation analysis generates 2D spectra, which identify spectral peaks that change 
either in phase (synchronous) or out of phase (asynchronous) [45]. Figure 3c shows an example of the power of this technique: the black line represents the 1D Raman spectrum that was derived from the 2D correlation analysis. This spectrum for isovaleric acid, measured upon uptake into the polymer from a gas-phase concentration of at most $617 \mathrm{ppm}_{\mathrm{v}}$ and obtained via $2 \mathrm{D}$ correlation analysis, matches very well with a reference Raman spectrum for isovaleric acid as bulk liquid (Figure 3c, red). Suitable agreement between the VOC and bulk reference Raman spectra was also observed over a larger spectral range, and for other relevant VOC biomarkers, such as 2amino-acetophenone (biomarker for P.aeruginosa), indole, and ethyl acetate (biomarkers for E.coli), as shown in Figure 4.

Each spectrum gives rise to clearly distinguishable sets of bands that can enable identification of these VOCs from complex mixture spectra. The VOC and reference spectra for the pure compounds (Figure 3c, Figure 4) have comparable signal-to-noise ratios, despite the much lower gas phase concentration of the compounds compared to bulk. This is particularly true for 2-aminoacetophenone and indole, which have a much lower vapor pressure than isovaleric acid, resulting in a gas phase concentration in the flow cell of at most 14 and $17 \mathrm{ppm}_{\mathrm{v}}$, respectively (see Supplementary Material). This concentration is $>10$ million fold lower than in bulk. No signal is detectable for analogous experiments performed without a polymer film on the sensor optic. This demonstrates the significant sensitivity improvement provided by concentrating the VOC in a thin polymer film in the evanescent excitation region of the sensor. Furthermore, the spectrum shown in Figure 4 bottom was obtained from the headspace of an aqueous solution containing a molar fraction of $\sim 2 \%$ ethyl acetate. No interference from water was observed, indicating that the polymer thin film effectively excludes water. This is 
corroborated by the finding that an analogous experiment conducted with pure water vapor resulted in only background (data not shown).

The method by which the polymer film is prepared impacts the sensor performance for VOC detection. All VOC spectra shown herein were obtained using drop cast polymer films that were allowed to dry and anneal for 1hour at room temperature prior to the VOC uptake experiment. Polymer films created in this manner give rise to some Raman peaks after 2D correlation analysis (Supplemental Material, Figure S2), likely due to slight conformational changes caused by continued polymer annealing. However, polymer peaks from films prepared in this manner do not dominate the VOC Raman spectra obtained after 2D correlation analysis (Figure 3c, Figure 4). Some but not all VOC spectra contain minor peaks that likely originate from the polymer. This may be due to specific interactions of these VOCs with the polymer matrix, which would lead to perturbations in the Raman bands of the polymer and the VOC. Indeed, such analytespecific perturbations have been observed in similar ATR-IR sensors [42], and can provide additional information, enabling the development of "smart sensors". For shorter annealing times, residual solvent peaks can be observed in the time series spectra and after $2 \mathrm{D}$ analysis. After overnight annealing at room temperature or at $\sim 40^{\circ} \mathrm{C}$, the Raman spectrum of the polymer films does not change appreciably over time, and 2D correlation analysis of these films reveals mainly noise. However, extensive drying decreases the sensitivity for VOC analysis, likely due to decreased uptake kinetics of VOCs from the gas phase into a more structured and dense polymer film.

For the experiments reported herein, the polymer thin film was irradiated at its center with $250 \mathrm{~mW}$ laser power for up to 4 hours, with continuous exposure if using a $3 \mathrm{~s}$ integration time, or exposure for $10 \mathrm{~s}$ every $30 \mathrm{~s}$ if using a $1 \mathrm{~s}$ integration time. Even continuous 4 hour laser 
irradiation of polymer thin films in this manner did not result in visible damage to the film. However, continuous irradiation may lead to film heating or non-visible damage to the film, which might change VOC absorption behavior and degrade the Raman spectra. To assess such potential confounding effects, we saturated a film with 2-aminoacetophenone vapor for 15 hours in the flow cell, then removed the VOC source. This film was then irradiated continuously with the laser at $250 \mathrm{~mW}$, and spectra were recorded every $30 \mathrm{~s}$. We observed no significant changes in the Raman peak positions and the relative peak intensities of 2-aminoacetophenone. However, the background subtracted Raman peak intensities for 2-aminoacetophenone increased by $20 \%$ over the four hour period. This increase was likely caused by continued absorption of 2aminoacetophenone remaining in the flow cell into the film. No degradation in the quality of the Raman spectrum was observed.

The optical sensor system (Figure 1) is designed to optimally excite and collect the Raman signal from VOCs concentrated in the polymer film. To estimate the degree of sensitivity enhancement offered by the evanescence optics, we compared the Raman intensity obtained using this optical system with that obtained by a high efficiency Raman Vector Probe focused onto the polymer thin film. This comparison was carried out using 2-aminoacetophenone as the test VOC based on its strong identifying Raman peak at $708 \mathrm{~cm}^{-1}$ (Figure 4). A First Contact ${ }^{\mathbb{R}}$ polymer film on the ZnSe lens was exposed to 2-aminoacetophenone vapors for 5-6 days to saturate the polymer, and spectra of the VOC saturated film were then acquired using the evanescent optics system and the LSI Raman Vector Probe.

The peak at $708 \mathrm{~cm}^{-1}$ acquired with the evanescent wave Raman probe using a one second integration time was $\sim 30$ fold more intense than the equivalent peak acquired with the conventional Raman probe using a 10 second integration time (Figure 5). This sensitivity 
enhancement was calculated based on the difference in peak height or area, with good agreement between the two measures. Taking into account the difference in integration time, the total degree of enhancement is approximately 300:1. To detect any clear signal with the LSI Raman Vector probe required the extreme case of saturating the polymer film for 5-6 days with the VOC, plus a longer integration time. Under normal operating conditions, during the initial absorption of VOCs into the polymer, the signal would be undetectable with the conventional LSI Raman Vector probe.

Beyond the sensitivity enhancement attained by the optics, 2D correlation analysis of time series spectra further increases the overall sensitivity by enhancing the signal and suppressing background and noise. 2D correlation analysis also provides additional information through the off-diagonal peaks observable in the 2D Raman spectrum (Figure 6). For low intensity peaks that are correlated to a peak of higher intensity, cross correlation causes the off-diagonal peaks to stand out more clearly than the diagonal elements.

Furthermore, spreading the peaks over a two-dimensional plane facilitates the identification of specific VOC biomarkers within complex mixture spectra, which increases the overall specificity. The time dependence of VOC uptake into the polymer film provides another dimension that can be exploited to deconvolute mixture spectra, for example by analyzing segmented data blocks [47]. To demonstrate the ability of our system to identify VOCs within a mixture, we have acquired time series spectra for VOC uptake into the polymer film from the headspace of a mixture containing equal masses of ethyl acetate, isovaleric acid, and 2-amino acetophenone. 
In the early time interval from $0-5$ minutes (Figure 7 and Figure 8 top), Raman spectra obtained via 2D correlation analysis are dominated by ethyl acetate absorption into the polymer film, as indicated by prominent peaks specific for this VOC (Table 1). In this same time window, a small amount of isovaleric acid is also detectable. In addition, the 2D analysis clearly resolves the $846 \mathrm{~cm}^{-1}$ ethyl acetate peak from the $836 \mathrm{~cm}^{-1}$ isovaleric acid peak. These two peaks are not resolved in the bulk spectrum, but are distinguishable through 2D correlation analysis. This distinction is noticeable in the 1D diagonal, but clearly evident in the 2D spectrum, and is likely due to the difference in adsorption kinetics. Therefore, 2D Raman correlation analysis improves the ability to resolve mixture spectra.

Ethyl acetate is the most volatile compound in the mixture and has the fastest absorption and desorption kinetics. Once the small amount of ethyl acetate introduced into the system is depleted in the input stream, the ethyl acetate desorbs from the thin film, leading to a decrease in ethyl acetate peak intensity in the time segment from 5-30 minutes. The decrease is clearly noticeable in the $2 \mathrm{D}$ off-diagonal peaks for the Raman band at $634 \mathrm{~cm}^{-1}$. A decreasing band gives rise to negative off-diagonal peaks in the synchronous spectrum, shown in blue. The ethyl acetate peaks are completely absent in the late time segment from 60-120 minutes. The 1D diagonal and 2D correlation spectra for the 5-30 minutes time segment (Figure 7 and Figure 8 center) are dominated by the spectral bands of isovaleric acid (Table 1). The spectral features of 2-amino acetophenone are noticeably growing in during the 5-30 minutes time segment, and dominate the spectrum in the 60-120 minutes time segment (Figure 7 and Figure 8 bottom). This demonstrates that our system can detect and differentiate VOCs present at low ppm concentrations as pure compounds and in mixtures.

\section{Conclusions}


We have designed, implemented, and demonstrated proof-of-principle for a novel Raman system that detects VOCs from the gas phase, based on robust and readily manufacturable evanescent wave optics. The system exhibits significantly higher sensitivity than conventional Raman probes, and enables VOC detection in the low-ppm range. This sensitivity enhancement is based on evanescent wave excitation of VOCs adsorbed and enriched in a thin polymer film, high collection efficiency provided by the optical design, and advanced data analysis methods that effectively suppress background features and noise. This preliminary proof of principle demonstration utilized a readily available commercial lens cleaning polymer. We expect that the system's sensitivity can be further increased into the ppb range through our planned efforts to investigate different polymers that provide optimal VOC enrichment in the film. Such a goal appears attainable, since Flavin et al. have demonstrated ppb level detection of p-nitro chlorobenzene, absorbed from water into a phenyl trimethyl silane film on a similar ATR-IR sensor [43].

By employing 2-D correlation analysis, we are able to readily identify and distinguish individual components in VOC mixtures, aided by their differential rates of adsorption into the polymer film. In future efforts, we aim to achieve automated VOC identification by creating a reference library of 2-D Raman spectral maps for targeted VOC biomarkers. These compounds can then be identified in other 2-D Raman spectra via fingerprint matching against this reference library of 2D Raman spectra, using a robust search algorithm.

In addition, compared to the bulk reference spectra, 2D correlation analysis also identified subtle changes in the polymer and VOC Raman spectra that are likely due to VOC-polymer interactions [42]. In future work, we will examine the nature of these Raman shifts for targeted VOCs as a function of sensing film polymer chemistry, to enable "smart sensing" that can 
provide additional selectivity, and thus may be used to enhance the identification of VOCs present in complex mixtures. Our ultimate goal is to apply these types of sensors to different medical, industrial, and biodefense applications.

\section{Acknowledgements}

Funding for this project was provided by the National Science Foundation through the Partnerships for Innovation awards IIP1237916. 


\section{References}

[1] M. Lechner and J. Rieder, Mass spectrometric profiling of low-molecular-weight volatile compounds - Diagnostic potential and latest applications, Curr. Med. Chem., 14 (2007) 987-995.

[2] C.D. Cox and J. Parker, Use of 2-Aminoacetophenone Production in Identification of Pseudomonas-Aeruginosa, J. Clin. Microbiol., 9 (1979) 479-484.

[3] J.J. Zhu, H.D. Bean, Y.M. Kuo, and J.E. Hill, Fast Detection of Volatile Organic Compounds from Bacterial Cultures by Secondary Electrospray Ionization-Mass Spectrometry, J. Clin. Microbiol., 48 (2010) 4426-4431.

[4] R.M.S. Thorn, D.M. Reynolds, and J. Greenman, Multivariate analysis of bacterial volatile compound profiles for discrimination between selected species and strains in vitro, J. Microbiol. Methods, 84 (2011) 258-264.

[5] W. Carroll, W. Lenney, T.S. Wang, P. Spanel, A. Alcock, and D. Smith, Detection of volatile compounds emitted by Pseudomonas aeruginosa using selected ion flow tube mass spectrometry, Pediatr. Pulmonol., 39 (2005) 452-456.

[6] M. O'Hara and C.A. Mayhew, A preliminary comparison of volatile organic compounds in the headspace of cultures of Staphylococcus aureus grown in nutrient, dextrose and brain heart bovine broths measured using a proton transfer reaction mass spectrometer, Journal of Breath Research, 3 (2009).

[7] G. Preti, E. Thaler, C.W. Hanson, M. Troy, J. Eades, and A. Gelperin, Volatile compounds characteristic of sinus-related bacteria and infected sinus mucus: Analysis by solid-phase microextraction and gas chromatography-mass spectrometry, Journal of Chromatography B-Analytical Technologies in the Biomedical and Life Sciences, 877 (2009) 2011-2018.

[8] M. Phillips, Method for the collection and assay of volatile organic compounds in breath, Anal. Biochem., 247 (1997) 272-278.

[9] M. Phillips, V. Basa-Dalay, G. Bothamley, R.N. Cataneo, P.K. Lam, M.P.R. Natividad, P. Schmitt, and J. Wai, Breath biomarkers of active pulmonary tuberculosis, Tuberculosis, 90 (2010) $145-151$.

[10] A.D. Wilson and M. Baietto, Advances in Electronic-Nose Technologies Developed for Biomedical Applications, Sensors, 11 (2011) 1105-1176.

[11] N. Guernion, N.M. Ratcliffe, P.T.N. Spencer-Phillips, and R.A. Howe, Identifying bacteria in human urine: Current practice and the potential for rapid, near-patient diagnosis by sensing volatile organic compounds, Clin. Chem. Lab. Med., 39 (2001) 893-906.

[12] M. Shnayderman, B. Mansfield, P. Yip, H.A. Clark, M.D. Krebs, S.J. Cohen, J.E. Zeskind, E.T. Ryan, H.L. Dorkin, M.V. Callahan, T.O. Stair, J.A. Gelfand, C.J. Gill, B. Hitt, and C.E. Davis, Species-specific bacteria identification using differential mobility spectrometry and bioinformatics pattern recognition, Anal. Chem., 77 (2005) 5930-5937. 
[13] J.E. Szulejko, M. McCulloch, J. Jackson, D.L. Mckee, J.C. Walker, and T. Solouki, Evidence for Cancer Biomarkers in Exhaled Breath, Ieee Sensors Journal, 10 (2010) 185210.

[14] W. Miekisch, J.K. Schubert, and G.F.E. Noeldge-Schomburg, Diagnostic potential of breath analysis - focus on volatile organic compounds, Clin. Chim. Acta, 347 (2004) 25-39.

[15] A.W. Jones and L. Andersson, Determination of ethanol in breath for legal purposes using a five-filter infrared analyzer: studies on response to volatile interfering substances, Journal of Breath Research, 2 (2008).

[16] C.D. Mansfield, H.H. Mantsch, and H.N. Rutt, Application of infrared spectroscopy in the measurement of breath trace compounds: A review, Canadian Journal of Analytical Sciences and Spectroscopy, 47 (2002) 14-28.

[17] J.K. Park, H.J. Yee, K.S. Lee, W.Y. Lee, M.C. Shin, T.H. Kim, and S.R. Kim, Determination of breath alcohol using a differential-type amperometric biosensor based on alcohol dehydrogenase, Anal. Chim. Acta, 390 (1999) 83-91.

[18] E. Kawakami, R.S. Machado, M. Reber, and F.R.S. Patricio, C-13-urea breath test with infrared spectroscopy for diagnosing Helicobacter pylori infection in children and adolescents, J. Pediatr. Gastroenterol. Nutr., 35 (2002) 39-43.

[19] S.P. Mondal, P.K. Dutta, G.W. Hunter, B.J. Ward, D. Laskowski, and R.A. Dweik, Development of high sensitivity potentiometric $\mathrm{NO}(\mathrm{x})$ sensor and its application to breath analysis, Sensors and Actuators B-Chemical, 158 (2011) 292-298.

[20] J.H. Shorter, D.D. Nelson, J.B. McManus, M.S. Zahniser, S.R. Sama, and D.K. Milton, Clinical study of multiple breath biomarkers of asthma and COPD (NO, CO(2), $\mathrm{CO}$ and $\mathrm{N}(2) \mathrm{O}$ ) by infrared laser spectroscopy, Journal of Breath Research, 5 (2011).

[21] B. Fruhberger, N. Stirling, F.G. Grillo, S. Ma, D. Ruthven, R.J. Lad, and B.G. Frederick, Detection and quantification of nitric oxide in human breath using a semiconducting oxide based chemiresistive microsensor, Sensors and Actuators B-Chemical, 76 (2001) 226-234.

[22] M. Moens, A. Smet, B. Naudts, J. Verhoeven, M. Ieven, P. Jorens, H.J. Geise, and F. Blockhuys, Fast identification of ten clinically important micro-organisms using an electronic nose, Lett. Appl. Microbiol., 42 (2006) 121-126.

[23] P. Montuschi, N. Mores, A. Trove, C. Mondino, and P.J. Barnes, The Electronic Nose in Respiratory Medicine, Respiration, 85 (2012) 72-84.

[24] S.H. Lim, S. Mix, Z.Y. Xu, B. Taba, I. Budvytiene, A.N. Berliner, N. Queralto, Y.S. Churi, R.S. Huang, M. Eiden, R.A. Martino, P. Rhodes, and N. Banaei, Colorimetric Sensor Array Allows Fast Detection and Simultaneous Identification of Sepsis-Causing Bacteria in Spiked Blood Culture, J. Clin. Microbiol., 52 (2014) 592-598. 
[25] K.S. Suslick, N.A. Rakow, and A. Sen, Colorimetric sensor arrays for molecular recognition, Tetrahedron, 60 (2004) 11133-11138.

[26] M. Moskovits, Surface-enhanced Raman spectroscopy: a brief retrospective, Journal of Raman Spectroscopy, 36 (2005) 485-496.

[27] K. Kneipp, H. Kneipp, and J. Kneipp, Surface-enhanced Raman scattering in local optical fields of silver and gold nanoaggregatess - From single-molecule Raman spectroscopy to ultrasensitive probing in live cells, Accounts of Chemical Research, 39 (2006) 443-450.

[28] E.C. Le Ru, P.G. Etchegoin, and M. Meyer, Enhancement factor distribution around a single surface-enhanced Raman scattering hot spot and its relation to single molecule detection, J. Chem. Phys., 125 (2006).

[29] S.M. Mansfield and G.S. Kino, Solid Immersion Microscope, Applied Physics Letters, 57 (1990) 2615-2616.

[30] C.A. Michaels, Surface-sensitive Raman microscopy with total internal reflection illumination, Journal of Raman Spectroscopy, 41 (2010) 1380-1387.

[31] L. Dhooge, J.M. Vigoureux, and C. Menu, General-Theory of the Raman-Scattering Close to A Plane Surface - Evanescent Raman-Spectra, J. Chem. Phys., 74 (1981) 3639-3659.

[32] P.R. Greene and C.D. Bain, Total Internal Reflection Raman Spectroscopy, Spectroscopy Europe, 16 (2004) 8-15.

[33] P.R. Greene and C.D. Bain, Total internal reflection Raman spectroscopy of barley leaf epicuticular waxes in vivo, Colloids and Surfaces B-Biointerfaces, 45 (2005) 174-180.

[34] Z.S. Nickolov, J.C. Earnshaw, and J.J. Mcgarvey, Total Internal-Reflection RamanSpectroscopy As A Method to Study Water-Structure Near Langmuir-Blodgett-Films, Journal of Raman Spectroscopy, 24 (1993) 411-416.

[35] A. Gonzalvez, S. Garrigues, M. de la Guardia, and S. Armenta, The ways to the trace level analysis in infrared spectroscopy, Analytical Methods, 3 (2011) 43-52.

[36] B. Pejcic, M. Myers, and A. Ross, Mid-Infrared Sensing of Organic Pollutants in Aqueous Environments, Sensors, 9 (2009) 6232-6253.

[37] P.T. Lin, V. Singh, J.J. Hu, K. Richardson, J.D. Musgraves, I. Luzinov, J. Hensley, L.C. Kimerling, and A. Agarwal, Chip-scale Mid- Infrared chemical sensors using air-clad pedestal silicon waveguides, Lab on A Chip, 13 (2013) 2161-2166.

[38] B. Murphy, P. Kirwan, and P. Mcloughlin, Study of the impact of penetrant characteristics upon diffusion into Teflon membranes to further assess the performance of an ATR/FTIR sensor, Analytical and Bioanalytical Chemistry, 377 (2003) 195-202. 
[39] R. Howley, B.D. MacCraith, K. O'Dwyer, H. Masterson, P. Kirwan, and P. Mcloughlin, Determination of hydrocarbons using sapphire fibers coated with poly(dimethylsiloxane), Applied Spectroscopy, 57 (2003) 400-406.

[40] K. Flavin, H. Hughes, V. Dobbyn, P. Kirwan, K. Murphy, H. Steiner, B. Mizaikoff, and P. Mcloughlin, A comparison of polymeric materials as pre-concentrating media for use with ATR/FTIR sensing, Int. J. Environ. Anal. Chem., 86 (2006) 401-415.

[41] R.P. Mccue, J.E. Walsh, F. Walsh, and F. Regan, Modular fibre optic sensor for the detection of hydrocarbons in water, Sensors and Actuators B-Chemical, 114 (2006) 438444.

[42] K. Flavin, H. Hughes, and P. Mcloughlin, The development of a novel smart mid-infrared sensing methodology for residual solvents, Int. J. Environ. Anal. Chem., 87 (2007) 29-42.

[43] K. Flavin, J. Mullowney, B. Murphy, E. Owens, P. Kirwan, K. Murphy, H. Hughes, and P. Mcloughlin, The development of novel organically modified sol-gel media for use with ATR/FTIR sensing, Analyst, 132 (2007) 224-229.

[44] I. Noda and O. Yukihiro, Two-Dimensional Correlation Spectroscopy - Applications in Vibrational and Optical Spectroscopy, John Wiley and Sons, New York, 2004.

[45] I. Noda, Generalized 2-Dimensional Correlation Method Applicable to Infrared, Raman, and Other Types of Spectroscopy, Applied Spectroscopy, 47 (1993) 1329-1336.

[46] I. Noda, Two-dimensional correlation analysis useful for spectroscopy, chromatography, and other analytical measurements, Analytical Sciences, 23 (2007) 139-146.

[47] I. Noda, Frontiers of Two-Dimensional Correlation Spectroscopy. Part 1. New concepts and noteworthy developments, Journal of Molecular Structure, 1069 (2014) 3-22.

[48] I. Noda, Frontiers of two-dimensional correlation spectroscopy. Part 2. Perturbation methods, fields of applications, and types of analytical probes, Journal of Molecular Structure, 1069 (2014) 23-49.

[49] I. Noda, Two-Dimensional Infrared-Spectroscopy, J. Am. Chem. Soc., 111 (1989) 81168118.

[50] H.J. Lai and P.Y. Wu, Hydration capabilities and structures of carbonyl and ether groups in poly(3-(2-methoxyethyl)-N-vinyl-2-pyrrolidone) film, Polymer Chemistry, 4 (2013) 33233332.

[51] P. Musto, G. Mensitieri, M. Lavorgna, G. Scarinzi, and G. Scherillo, Combining Gravimetric and Vibrational Spectroscopy Measurements to Quantify First- and SecondShell Hydration Layers in Polyimides with Different Molecular Architectures, Journal of Physical Chemistry B, 116 (2012) 1209-1220. 
[52] Y.L. Yang, W. Yan, and C.Y. Jing, Dynamic Adsorption of Catechol at the Goethite/Aqueous Solution Interface: A Molecular-Scale Study, Langmuir, 28 (2012) 14588-14597.

[53] H.J. Lai, Z.W. Wang, P.Y. Wu, B.I. Chaudhary, S.S. Sengupta, J.M. Cogen, and B. Li, Structure and Diffusion Behavior of Trioctyl Trimellitate (TOTM) in PVC Film Studied by ATR-IR Spectroscopy, Industrial \& Engineering Chemistry Research, 51 (2012) 93659375.

[54] Y.C. Fu and L.T. Lim, Investigation of multiple-component diffusion through LLDPE film using an FTIR-ATR technique, Polymer Testing, 31 (2012) 56-67.

[55] B. Muik, B. Lendl, A. Molina-Diaz, M. Valcarcel, and M.J. Ayora-Canada, Twodimensional correlation spectroscopy and multivariate curve resolution for the study of lipid oxidation in edible oils monitored by FTIR and FT-Raman spectroscopy, Anal. Chim. Acta, 593 (2007) 54-67.

[56] A. Jirasek, H.G. Schulze, C.H. Hughesman, A.L. Creagh, C.A. Haynes, M.W. Blades, and R.F.B. Turner, Discrimination between UV radiation-induced and thermally induced spectral changes in AT-paired DNA oligomers using UV resonance Raman spectroscopy, Journal of Raman Spectroscopy, 37 (2006) 1368-1380.

[57] V.A. Shashilov and I.K. Lednev, Two-dimensional correlation Raman spectroscopy for characterizing protein structure and dynamics, Journal of Raman Spectroscopy, 40 (2009) 1749-1758.

[58] H. Tang, S.T. Sun, J.W. Wu, P.Y. Wu, and X.H. Wan, Conformational changes in novel thermotropic liquid crystalline polymer without conventional mesogens: A Raman spectroscopic investigation, Polymer, 51 (2010) 5482-5489.

[59] Y.Z. Ren, T. Murakami, T. Nishioka, K. Nakashima, I. Noda, and Y. Ozaki, Twodimensional Fourier transform Raman correlation spectroscopy studies of polymer blends: Conformational changes and specific interactions in blends of atactic polystyrene and poly(2,6-dimethyl-1,4-phenylene ether), Macromolecules, 32 (1999) 6307-6318.

[60] S.H. Yalkowsky and R.M. Dannenfelser, Aquasol Database of Aqueous Solubility, College of Pharmacy, University of Arizona, Tucson, AZ, 1992.

[61] J. Zhao, H. Lui, D.I. Mclean, and H. Zeng, Automated autofluorescence background subtraction algorithm for biomedical Raman spectroscopy, Applied Spectroscopy, 61 (2007) 1225-1232.

[62] Y.M. Jung, Principal component analysis based two-dimensional correlation spectroscopy for noise filtering effect, Vibrational Spectroscopy, 36 (2004) 267-270.

[63] J. Mertz, Radiative absorption, fluorescence, and scattering of a classical dipole near a lossless interface: a unified description, Journal of the Optical Society of America BOptical Physics, 17 (2000) 1906-1913. 
[64] S.L. Chuang, Physics of Photonic Devices, John Wiley and Sons, New York, 2009.

[65] L.D.J. Bos, P.J. Sterk, and M.J. Schultz, Volatile Metabolites of Pathogens: A Systematic Review, Plos Pathogens, 9 (2013).

[66] W. Filipiak, A. Sponring, M.M. Baur, A. Filipiak, C. Ager, H. Wiesenhofer, M. Nagl, J. Troppmair, and A. Amann, Molecular analysis of volatile metabolites released specifically by staphylococcus aureus and pseudomonas aeruginosa, Bmc Microbiology, 12 (2012). 


\section{Figure Legends}

Figure 1. Sensor optics: Ray tracing scheme (top) and actual system (bottom). (a) Filtered laser light is reflected from (b) an adjustable gold mirror, and (c) a dichroic mirror, prior to being focused by (d) an aspheric lens onto (e) a ZnSe hemisphere. Scattered Raman emission is collimated by the aspheric lens, then passed through the dichroic mirror and focused by (f) an achromatic doublet onto $(\mathrm{g})$ the fiber bundle that transmits the output signal onto the $\mathrm{CCD}$ detector in the instrument. In the actual system, the orientation of the aspheric lens and $\mathrm{ZnSe}$ hemisphere is rotated by $90^{\circ}$ by another mirror so that the sensor surface is horizontal. A flow cell is mounted on top of the stage holding the $\mathrm{ZnSe}$ hemisphere.

Figure 2. Key optical components of the evanescent sensor probe, enabling excitation at the critical angle $\Theta_{c}$, and optimal collection of the Raman emission signal. The calculated [63] anisotropic Raman emission intensity distribution is indicated inside the schematic of the hemisphere sensor lens.

Figure 3. (a) Time-dependent uptake of isovaleric acid as gas phase VOC into a thin polymer film on top of the Raman sensor, measured via changes in Raman intensity at its peak position. (b) Selected Raman spectra from this time series (10 spectra in 2 minute intervals, up to 20 minutes), showing increasing isovaleric acid peaks and a decreasing baseline consisting mainly of polymer bands. (c) Normalized stack plot with 1D diagonal spectrum obtained after synchronous 2D correlation analysis of the time series VOC uptake data (black), showing good agreement with a bulk liquid reference Raman spectrum of isovaleric acid (red).

Figure 4. Raman spectra obtained for example biomarker VOCs after synchronous 2D correlation analysis (black), compared to bulk reference Raman spectra (red). The reference spectra of isovaleric acid, 2-aminoacetophenone, and indole were obtained from the pure compounds. The spectrum of ethyl acetate was obtained from the headspace of ethyl acetate dissolved in water at a $\sim 2 \%$ molar fraction. 
Figure 5. Raman spectra obtained from a polymer thin film saturated with 2aminoacetophenone, using the evanescent optics system (EV-Optics) with 1 second integration time, and a high efficiency LSI Raman Vector Probe (RVP) with 10 second integration time.

Figure 6. Synchronous 2D Raman spectrum of isovaleric acid as VOC adsorbed into a thin polymer film, with 1D diagonal spectrum shown on top. The lower intensity peaks at 879,904 , and $962 \mathrm{~cm}^{-1}$ show up more clearly in the off-diagonal elements created through cross correlation with the high intensity peak at $836 \mathrm{~cm}^{-1}$, compared to the diagonal elements which are based on autocorrelation.

Figure 7. Raman spectra obtained from a VOC mixture containing ethyl acetate, isovaleric acid, and 2-aminoacetophenone. (a) VOC 1D diagonal spectra obtained after segmented synchronous 2D correlation analysis of separate data blocks corresponding to intervals within the time series, as indicated in the graph. (b) Conventional bulk reference Raman spectrum of the liquid mixture. Vertical lines and symbols at the top indicate the position of Raman bands specific for ethyl acetate (blue diamond), isovaleric acid (red triangle), and 2-aminoacetophenone (green circle). See Table 1 for a listing of the peak positions.

Figure 8. Synchronous 2D Raman spectra obtained from a VOC mixture containing ethyl acetate, isovaleric acid, and 2-aminoacetophenone, adsorbed into a thin polymer film. Segmented analysis of separate data blocks corresponding to intervals within the time series: $0-5$ minutes (top), 5-30 minutes (center), and 60-120 minutes (bottom). Symbols indicate the position of Raman bands specific for ethyl acetate (blue diamond), isovaleric acid (red triangle), and 2aminoacetophenone (green circle). 
Table 1: Prominent Raman Peaks for Targeted VOCs in the range of $500-1000 \mathrm{~cm}^{-1}$.

\begin{tabular}{cc}
\hline Compound & Peak Position $\left[\mathrm{cm}^{-1}\right]$ (Normalized Intensity) \\
\hline Ethyl Acetate & VOC: $634(1) \mid 846(0.43)$ \\
& Bulk: $633(1) \mid 846(0.60)$ \\
Isovaleric Acid & VOC: $836(1)|962(0.44)| 879(0.26) \mid 904(0.16)$ \\
& Bulk: $836(1)|962(0.37)| 883(0.22) \mid 905(0.17)$ \\
2-Amino Acetophenone & VOC: $708(1)|561(0.59)| 841(0.24)$ \\
& Bulk: $708(1)|561(0.36)| 841(0.17)$ \\
Indole & VOC: $759(1)|543(0.38)| 608(0.36)$ \\
& Bulk: $760(1)|545(0.45)| 608(0.20)$ \\
\hline
\end{tabular}


Figure 1:
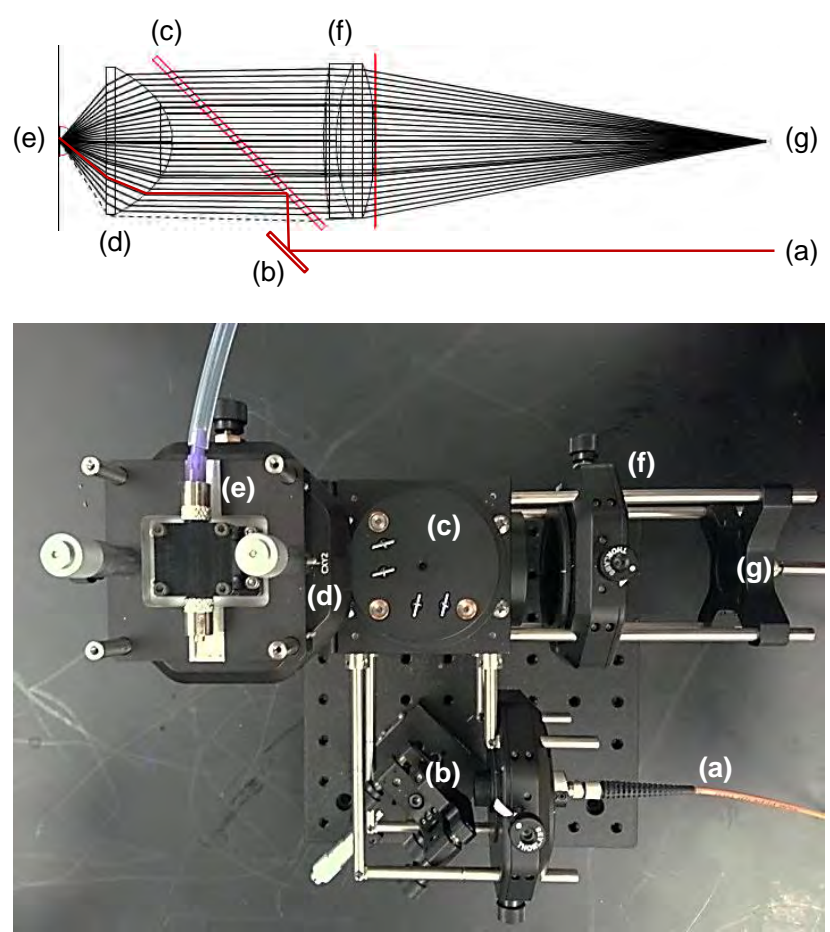
Figure 2:

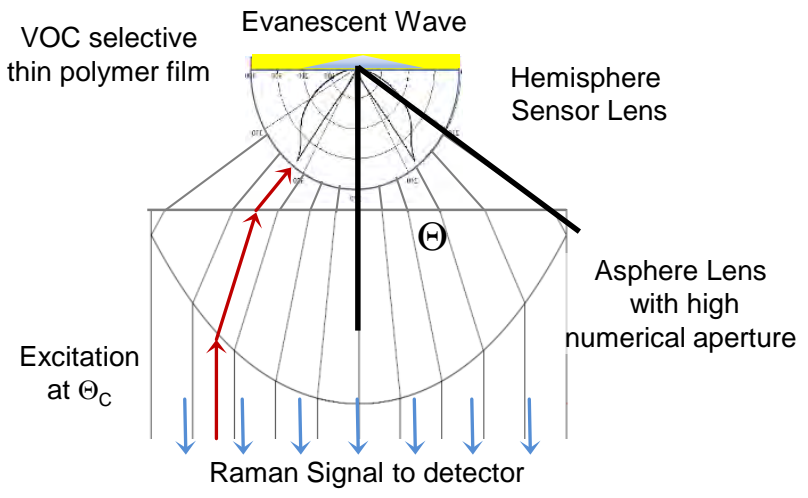


Figure 3:
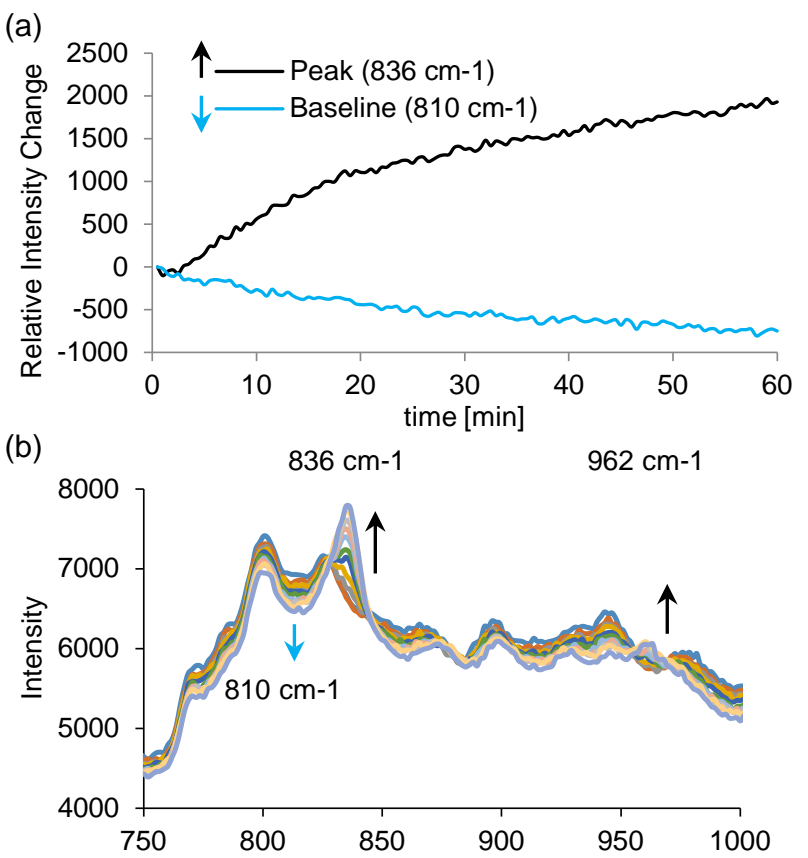

(c)

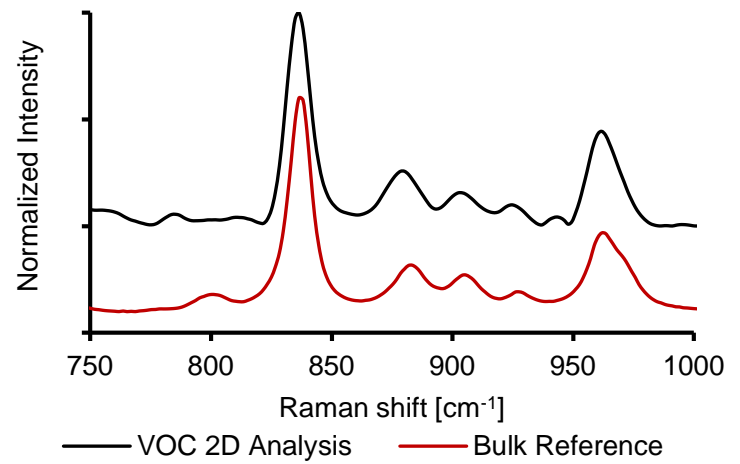


Figure 4:
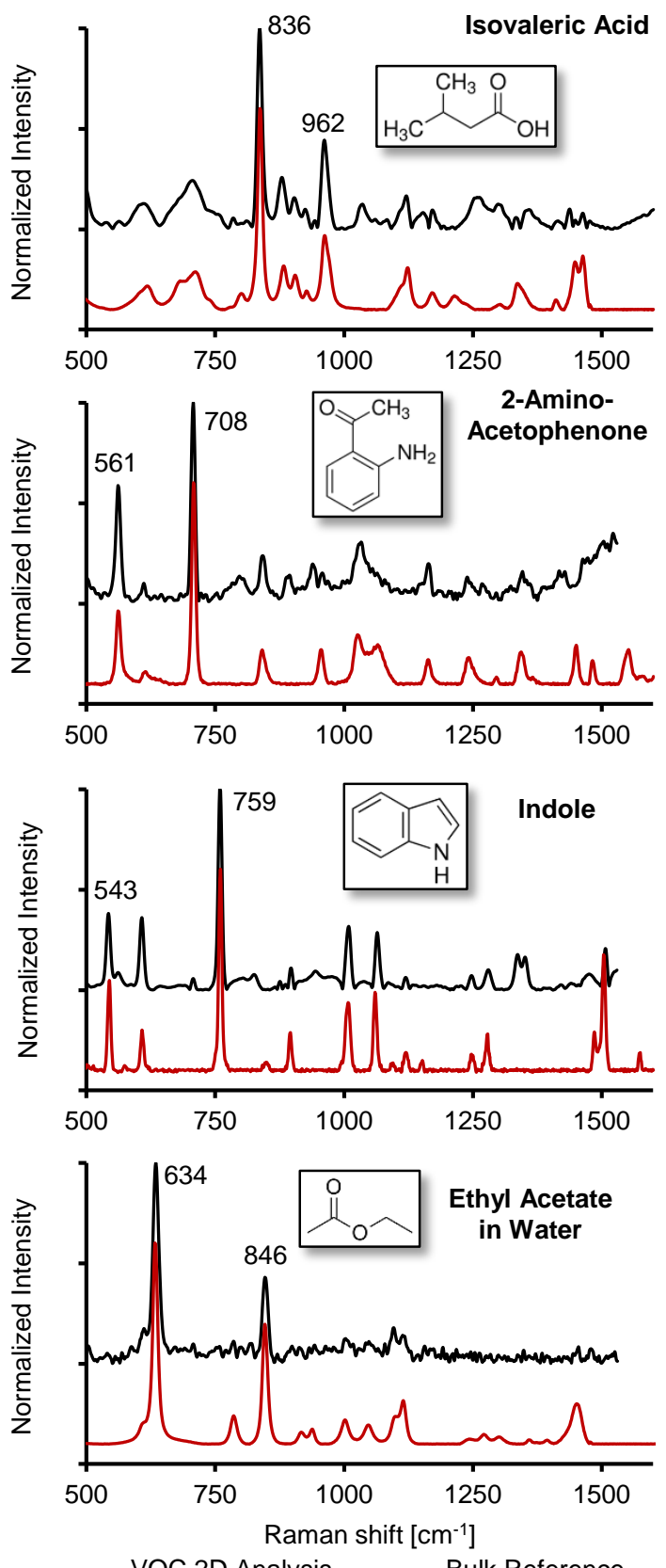
Figure 5:

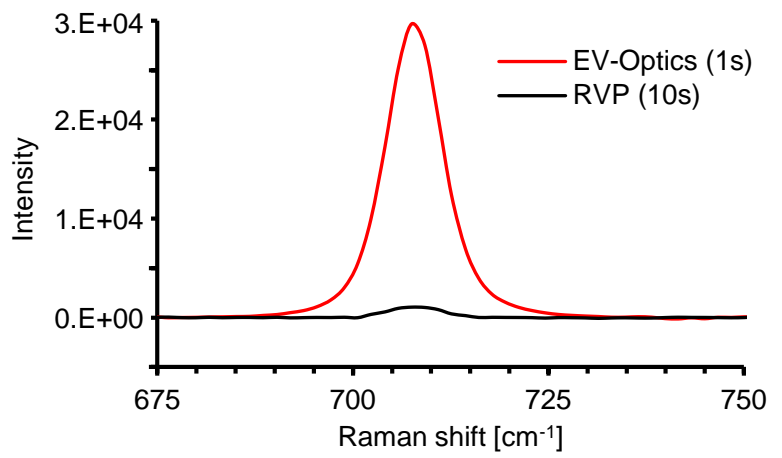


Figure 6:

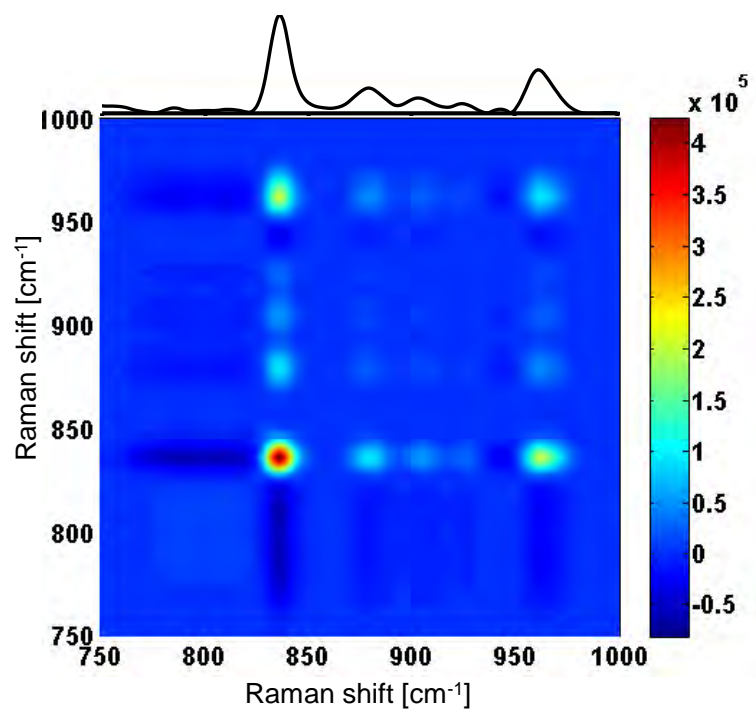


Figure 7:
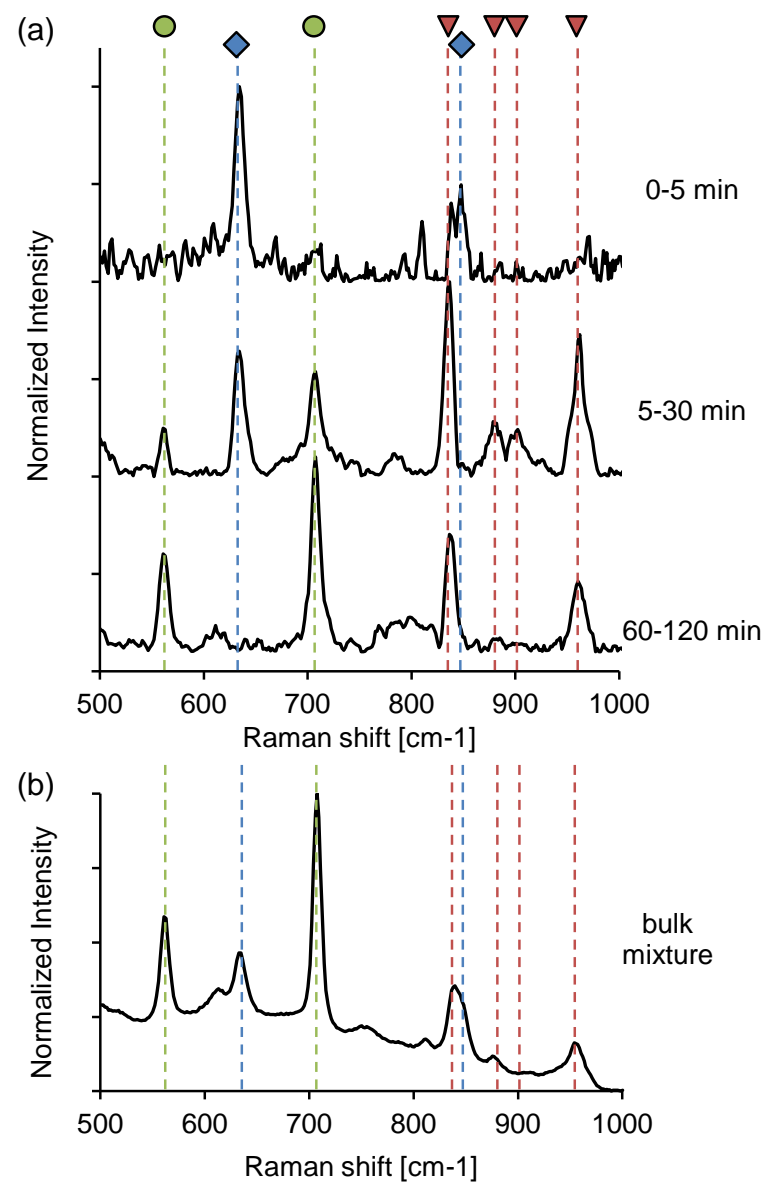
Figure 8:

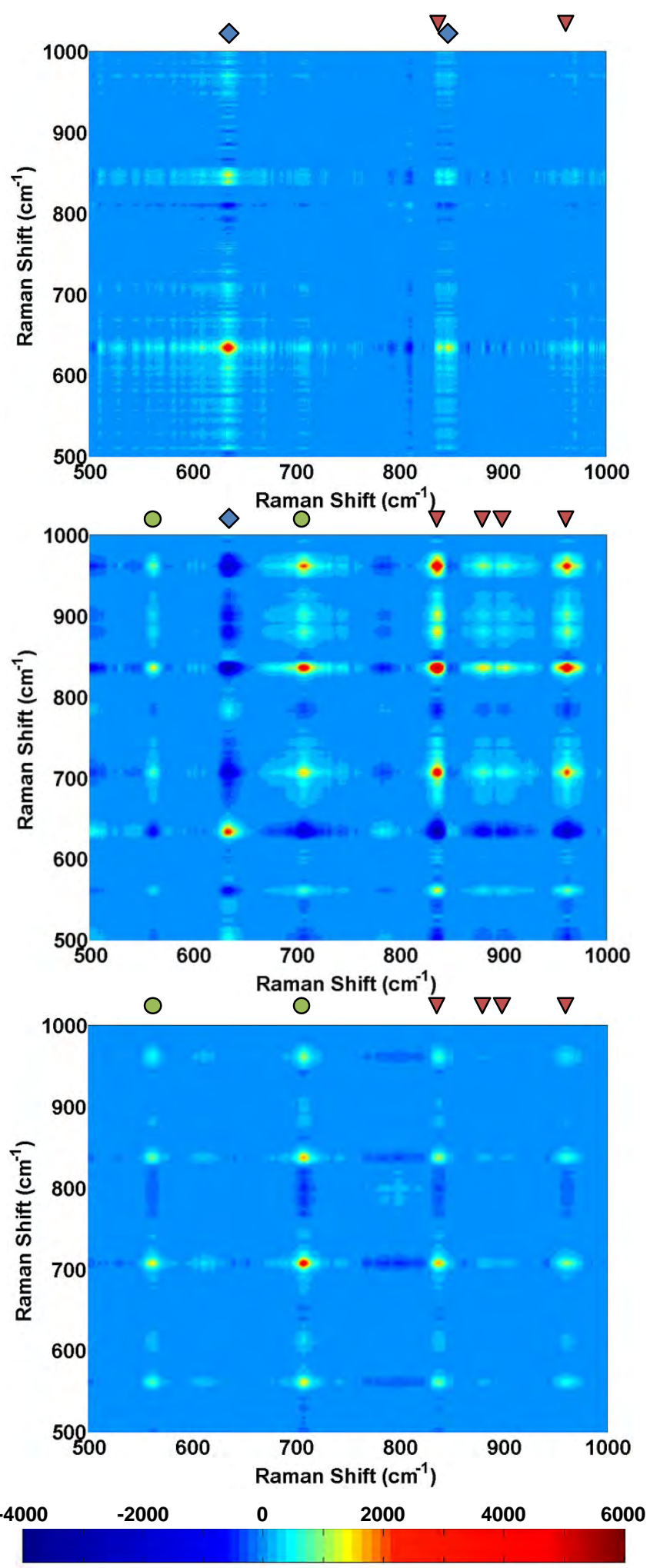

